\title{
Estimation de la rentabilité de la culture de certains eucalyptus dans le sud-ouest de la France
}

\author{
Jean-Philippe Terreaux* \\ IGREF, Cemagref, BP. 5095, 34033 Montpellier Cedex 1, France
}

(Reçu le 12 novembre 1998; accepté le 2 septembre 1999)

\begin{abstract}
Résumé - L'objectif de ce travail est de donner des indications sur l'intérêt économique, pour des propriétaires fonciers dans le sudouest de la France, de la plantation d'eucalyptus, une essence attrayante par les qualités du bois et la rapidité de croissance des arbres, mais soumise à un risque de gel qu'il est impossible de négliger dans la prise de décision. Dans un premier temps nous donnons les raisons de l'emploi de certains critères de gestion dans un contexte aléatoire : espérance de revenu, revenu médian, la valeur du revenu telle qu'il y ait une certaine probabilité d'obtenir un résultat pire, ou d'obtenir un résultat meilleur, la probabilité d'avoir un revenu inférieur ou supérieure à une certaine valeur. Ensuite nous décrivons la méthode utilisée (méthode de Monte-Carlo) et nous déterminons l'horizon des calculs et le nombre de simulations nécessaires à l'obtention d'une précision donnée sur les résultats. Les résultats numériques sont donnés pour le gundal et le gunnii, pour des durées de retour du gel s'étalant entre10 et 30 ans, et des âges de coupe entre 9 et 13 ans. Il est ainsi possible de donner des indications pour la détermination de l'âge optimal de récolte des parcelles. En outre, en comparant les résultats issus soit de 10 ha de gundal, soit de 10 ha de gunnii, soit encore de 5 ha de chacune de ces espèces, nous donnons des éléments sur l'intérêt d'une diversification des plantations.
\end{abstract}

eucalyptus / gestion / économie / risque / Monte-Carlo

Abstract - Assessment of the profitability of the culture of eucalyptus in south-west of France. We determine some economic parameters related to the plantation in the south-west of France of eucalyptus, which has particularly good properties for pulp production, but which is the object of relatively frequent frost hazards. Firstly we define the management criteria which are suitable to guide the decider. Secondly we use a Monte-Carlo method to compute the value of these criteria for the E. gundal and E. gunnii, for harvest dates varying between 9 and 13 years, and the expectancy of the intervals between two destructive frost between 10 and 30 years. This requires before to determine the minimum time horizon of the simulations in order to have a sufficient precision, and the minimum number of simulations. We make also some developments on the diversification of risks, by supplying the results corresponding to ten hectares planted either with each of the two species, or either with five hectares of E. gundal and five ha of E. gunnii.

eucalyptus / management / economics / risk / Monte-Carlo

\section{INTRODUCTION}

L'eucalyptus, originaire d'Australie, est un arbre dont certaines espèces, en particulier l'E. gundal et l'E. gunnii, croissent rapidement tout en produisant un bois de qualité, sur des sols qui peuvent être médiocres, à l'exception des sols calcaires. De plus les arbres peuvent rejeter plusieurs fois de souche, permettant des économies substantielles de travaux de replantation.

Malheureusement, toutes les qualités de ces espèces sont contrebalancées par leur grande sensibilité au gel, sensibilité souvent d'autant plus grande que leur

* Correspondance et tirés à part

Tél. 04670463 49; Fax. 04676357 95; e-mail : jean-philippe.terreaux@cemagref.fr 
croissance est rapide. L'Association Forêt Cellulose AFOCEL - a d'ailleurs entrepris en France un travail important de sélection génétique sur ces caractéristiques de productivité et de résistance au gel.

La probabilité de gel pour une espèce donnée, dans un lieu donné, est relativement bien connue dans le sudouest de la France, grâce à une étude de Météo-France sur les caractéristiques de ces événements (température minimale atteinte, durée de la période froide, variation relative par rapport aux jours précédents...).

L'objet de ce travail est de déterminer les paramètres économiques d'une telle production, en fonction de la durée de retour du gel, de la loi de croissance de l'espèce étudiée, de l'âge de coupe des arbres et enfin de différentes données technico-économiques. Le gel étant un phénomène aléatoire, les revenus issus de la plantation d'eucalyptus le seront aussi. Nous déterminons ici l'espérance mathématique de chacun des critères économiques retenus.

Les résultats obtenus devraient permettre de mieux définir le programme de boisement qui sera engagé par la collaboration des propriétaires fonciers, des utilisateurs du bois et de la puissance publique.

Comme le risque de gel est porté en grande partie par les propriétaires fonciers, même s'il est envisagé de mettre en place un processus d'assurance, c'est de leur point de vue que nous évaluerons la rentabilité globale de cet investissement. Aussi nous définissons section 2.1 les critères de gestion utilisés.

En section 2.2, nous indiquons les raisons du choix de la méthode de calcul que nous utilisons (méthode de Monte Carlo), et nous indiquons comment certains paramètres ont été évalués.

Les résultats donnés dans la section 3 nous permettent en particulier de comparer la productivité de deux essences (l'E. gundal et l'E. gunnii), et de déterminer aussi jusqu'à quel point il est intéressant de diversifier les risques.

Bien entendu, ces résultats ne constituent qu'une aide à la décision, la détermination des risques qu'un agent économique est prêt à encourir pour augmenter la rentabilité de ses investissements restant de son seul ressort.

\section{MÉTHODE}

\subsection{Critères de choix en situation aléatoire}

Il existe de nombreux critères de décision en situation de risque ou d'incertitude. Chacun repose sur des hypothèses explicitement ou implicitement établis. Ainsi von Neuman et Morgenstern [9] ont démontré que les critères dits d'utilité espérée reposent sur trois hypothèses de rationalité des agents économiques. Toutefois, ces critères peuvent conduire à certaines contradictions empiriques (par exemple le paradoxe d'Allais, qui montre que l'une des trois hypothèses de rationalité n'est en fait pas toujours valable), contradictions que tente de corriger par exemple la théorie de l'utilité dépendant des rangs (RDU, [1]) ou d'autres critères dérivés. En fait ils sont d'un emploi difficile en pratique, puisque nécessitant l'estimation de différentes fonctions (la fonction d'utilité, la fonction de transformation des probabilités), supposées différer d'un agent économique à l'autre.

Il existe toute une famille d'autres critères, dont on connaît parfois assez mal les hypothèses sous-jacentes, mais qui ont pour mérite un emploi aisé, en particulier parce qu'ils n'impliquent pas cette estimation de fonctions ou de paramètres propres à chaque agent. On ne cherche plus alors une seule solution, supposée être optimale, mais on se contente d'éliminer les solutions qui sont dominées pour chacun des critères exposés. Cela conduit aussi à augmenter la connaissance qu'ont les agents économiques des risques encourus et des bénéfices attendus. Ayant une meilleure perception des risques, ces derniers peuvent alors prendre de meilleures décisions.

Différents critères peuvent ainsi être sélectionnés [2]. Ceux que nous présentons sont les plus couramment employés : face à une décision assujettie à un risque, c'est-à-dire face à une fonction de distribution de revenus aléatoires, le décideur va en général répartir en deux classes certains aspects de cette distribution : ce qui est désirable et ce qui ne l'est pas. Notons au préalable que tous ces paramètres correspondent ici à des calculs sur un horizon infini. Nous reviendrons sur cet aspect à la section 2.2.

Les paramètres « positifs » (au sens où on recherche à augmenter leur valeur) sont par exemple :

-l'espérance de gain;

- le gain médian (moins sensible aux valeurs extrêmes);

- le gain défini par le fait qu'il y a une certaine probabilité (par exemple $5 \%$ ) d'obtenir un gain pire;

- le gain défini par le fait qu'il y a une certaine probabilité d'obtenir un gain meilleur;

- la probabilité d'obtenir au moins un niveau minimum de gain.

Les paramètres « négatifs » sont par exemple :

-l'écart type des gains;

- le semi-écart type des valeurs inférieures à la moyenne, qui diffère du précédent lorsque la distribution des gains n'est pas symétrique, et lorsque le décideur est plus sensible à ce qui se passe pour des valeurs inférieures à la moyenne (risques de pertes) qu'aux 
valeurs supérieures à la moyenne (opportunités de gain);

- la probabilité d'obtenir un gain négatif ou inférieur à une certaine valeur (par exemple le coût d'opportunité du sol);

-l'espérance mathématique de perte, sous condition qu'il y ait une perte.

Quels que soient les paramètres «positifs » ou «négatifs » mesurés, on choisit, si cela est possible, la situation qui donne simultanément la valeur la plus grande aux paramètres positifs et la valeur la plus petite aux paramètres négatifs. Mais lorsqu'entre deux décisions possibles, leur issue se traduit par des différences de même signe de paramètres positifs et négatifs, ou des différences de signes contraires entre deux paramètres positifs, ou entre deux paramètres négatifs, on ne peut pas dire que l'une de ces décisions est préférable à l'autre. Le choix appartient au décideur.

Ici, pour des questions de facilité d'interprétation, on a choisi de calculer l'espérance et l'écart type du revenu (au sens du revenu actualisé sur un horizon infini ou suffisamment grand), ainsi que le revenu médian, la valeur du revenu telle qu'il y ait une probabilité de $5 \%$ d'obtenir un revenu pire, la valeur du revenu tel qu'il y ait une probabilité de $5 \%$ d'obtenir un revenu meilleur, la probabilité d'avoir un revenu inférieur à $10 \mathrm{kF}$ et enfin la probabilité d'avoir un revenu supérieur à $30 \mathrm{kF}$ pour le gundal et $20 \mathrm{kF}$ pour le gunnii (pour un hectare, sur un horizon infini, ces valeurs étant à comparer avec le prix du foncier : voir [11]). Dans la section sur la diversification, qui correspond à une plantation totale sur 10 ha, ces valeurs de $10 \mathrm{kF}$ et 20 ou $30 \mathrm{kF}$ sont remplacées respectivement par $100 \mathrm{kF}$ et $200 \mathrm{kF}$.

\subsection{Utilisation d'une méthode de Monte-Carlo}

\subsubsection{Principes de base}

La recherche des valeurs numériques prises par les critères sélectionnés précédemment est assez difficile sur le plan analytique. Aussi nous employons une méthode de Monte-Carlo, qui permet aisément d'en obtenir une estimation. Son principe consiste ici à simuler la réalisation d'événements gel ou de non-gel, sur un horizon $H$, avec les probabilités adaptées, et à en calculer les conséquences. On appelle ici simulation une suite de ces événements sur un horizon $H$. En répétant un grand nombre de fois ces opérations, on peut estimer l'espérance mathématique de chacun des critères retenus.

On comprend alors qu'il est nécessaire en premier lieu de déterminer l'horizon $H$ minimum et le nombre de simulations nécessaires pour avoir une estimation satisfaisante des «vraies » valeurs, sans trop allonger les temps de calcul.

\subsection{2. Évaluation de l'horizon et du nombre de simulations}

Trois problèmes sont à résoudre pour l'emploi de cette méthode.

1) Le premier est lié à l'horizon, nécessairement fini sur lequel on va travailler. Suite à la réalisation à des dates indéterminées de l'événement gel, il n'est pas possible de faire correspondre un horizon fini de travail à un nombre entier de rotations. Cela enlève toute possibilité d'un passage classique, selon la méthode de Faustmann [5], d'un horizon fini à un horizon infini.

La solution retenue consiste à travailler directement sur un horizon, noté $H$, suffisamment grand, pour que l'espérance de la valeur actualisée sur pied du peuplement à cet horizon soit négligeable (c'est-à-dire soit inférieure à $1 \%$, seuil de tolérance retenu) par rapport au bénéfice actualisé obtenu entre le présent et cet horizon.

En pratique, connaissant la valeur maximale sur pied du peuplement pour les deux espèces considérées, pour un âge de coupe donné, et suite au calcul d'une première approximation de l'espérance de revenu, avec un horizon $H^{\prime}$ suffisamment grand (il nous suffit ensuite de vérifier que $H^{\prime} \geq H$ ), on détermine une valeur de $H$, conduisant à un rapport inférieur à $1 \%$ entre d'une part cette valeur maximale du peuplement à la date $H$ et d'autre part l'espérance de revenu sur l'horizon $H^{\prime}$. Pour simplifier, on a mené ce calcul dans le cadre des différentes durées de retour du gel étudiées, et on a retenu pour l'ensemble des calculs réalisés ultérieurement la plus grande des valeurs de $H$ ainsi trouvées.

On peut alors transformer ce résultat correspondant à l'horizon fini $H$ en un résultat en horizon infini, par une correction à la Faustmann [5].

Soit $R_{H}$ l'espérance de revenu sur l'horizon $H$, suite à une chronique donnée, de durée $H$, des destructions de la parcelle. Le revenu sur un horizon infini, $R_{\propto}$, correspondant de fait à une répétition infinie de cette chronique, s'écrit :

$$
\begin{aligned}
R_{\infty} & =R_{H}\left[1+\frac{1}{(1+r)^{H}}+\frac{1}{(1+r)^{2 H}}+\ldots\right] \\
& =R_{H} \frac{(1+r)^{H}}{(1+r)^{H}-1} .
\end{aligned}
$$

Ce dernier résultat reste ainsi précis à $1 \%$ près. Mais on note que cette méthode amplifie les valeurs extrêmes 
de la distribution : supposons par exemple que pendant les $H$ premières années aucun gel n'ait eu lieu. La probabilité d'un tel événement est très faible. Or la transformation précédente suppose qu'aucun gel n'aura lieu non plus par la suite, situation d'une probabilité nulle. En pratique, les futures périodes de gel devraient ramener le résultat vers sa valeur moyenne. Mais comme ces événements auront lieu au delà de l'horizon $H$, l'actualisation rend cette correction négligeable en pratique.

Numériquement, le cas le plus défavorable est obtenu pour l'Eucalyptus gunnii, avec durée de retour du gel de 10 ans et coupe des arbres à 11 ans. $H$ est alors égal à 287 ans. Dans la suite des calculs, on prend $H=290$ ans.

2) Le second problème est la détermination du nombre de simulations nécessaires pour obtenir, sur cet horizon fini, un résultat de précision donnée.

Un premier majorant de ce nombre est due à l'utilisation de l'inégalité de Bienaymé-Tchébychev : on remarque en premier lieu, avec Hammersley et Handscomb [7], que le problème que nous avons à résoudre, ainsi que de nombreux autres problèmes permettant l'utilisation de méthode de Monte Carlo, reviennent numériquement à un calcul d'intégrale : en effet, le résultat obtenu est une fonction $R$ des différents résultats de simulation notés $x_{1}, x_{2} \ldots x_{n}$, et peut en conséquence s'interpréter comme un estimateur sans biais d'une intégrale multiple de cette fonction :

$$
\int_{0}^{1} \int_{0}^{1} \ldots \int_{0}^{1} R\left(x_{1}, x_{2}, \ldots x_{n}\right) \mathrm{d} x_{1} \mathrm{~d} x_{2} \mathrm{~d} x_{n}
$$

En utilisant ce résultat, et l'inégalité de BienayméTchébychev, on en déduit, avec Fishman [6], que le nombre de simulations, pour donner une valeur précise à $\varepsilon$ près, avec un niveau de confiance de $1-\delta$, est de : $n=1 /\left(4 \delta \varepsilon^{2}\right)$, soit, pour $\varepsilon=1 \%$ et $\delta=5 \%$ (soit une précision de $1 \%$ avec un niveau de confiance de $95 \%$ ), $n=50000$ simulations. Mais, comme l'inégalité sur laquelle il est fondé, ce résultat correspond au pire des cas possibles.

Si le résultat des simulations suivait une loi gaussienne, il serait aisé d'utiliser d'autres résultats de la théorie des probabilités afin de donner une estimation plus précise du nombre $n$ minimum de simulations permettant d'atteindre le résultats avec la précision recherchée (voir [13]).

Des tests effectués sur les résultats de 20000 simulations montrent que ces résultats n'ont malheureusement pas la propriété d'être gaussiens : sur l'espérance de gain par exemple, la moyenne diffère sensiblement de la médiane, comme en témoignent les résultats des tableaux I et II. La figure 1 le confirme en mettant en évidence la forme dissymétrique de la distribution des résul-

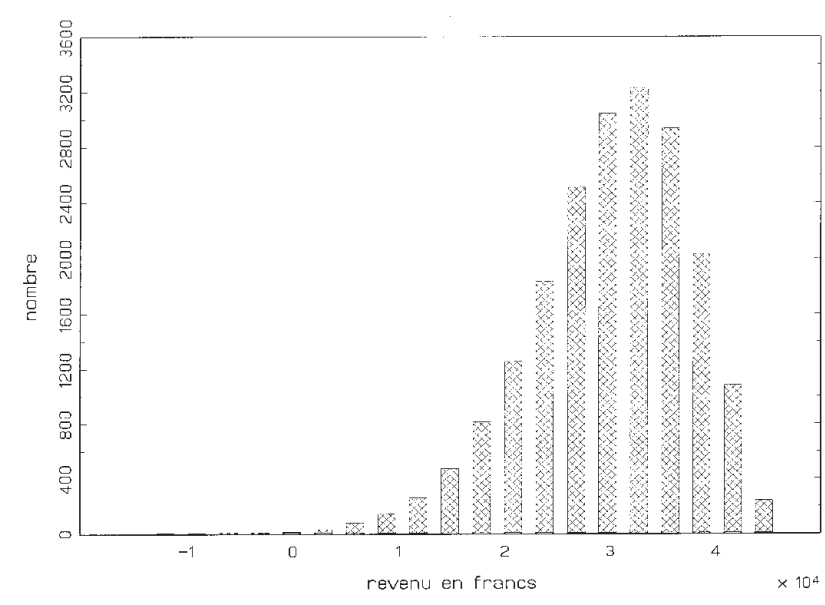

Figure 1. Eucalyptus gundal, durée de retour du gel de 15 ans, arbres coupés à 11 ans : distribution des revenus pour 20000 simulations.

tats (qui correspondent au gundal, avec une durée de retour du gel de 15 ans et une coupe des arbres à 11 ans; d'autres choix de paramètres conduisent à des courbes de même allure). Enfin un test du chi2 réalisé sur ces données conduit à un résultat négatif, à savoir que la probabilité de se tromper en affirmant que ces résultats ne sont pas gaussiens est inférieure à $10^{-6}$.

Une solution pragmatique consiste alors à effectuer un grand nombre de simulations (mais restant toutefois inférieur à 50000) et à constater à partir de quel nombre le résultat final (c'est-à-dire celui obtenu avec 50000 simulations) pour chacun des critères est approché avec une précision satisfaisante, à savoir ici meilleure que $1 \%$. En répétant plusieurs fois l'opération, pour différentes valeurs des paramètres et pour différents critères, on peut ainsi évaluer le nombre de simulations qui sera effectué lors de chacun des calculs suivants. La figure 2 illustre cette méthode en donnant l'évolution du résultat du critère « probabilité d'avoir un gain supérieur à $30000 \mathrm{~F}$ », pour le gundal avec une durée de retour du gel de 30 ans et un âge de coupe de 11 ans, en fonction du nombre de simulations utilisées dans les calculs. Comme l'illustre bien cette figure, il est nécessaire d'utiliser environ 20000 simulations pour obtenir la précision recherchée.

On notera que le résultat obtenu a pu être confirmé dans un cas simplifié (parcelle recépable indéfiniment) pour lequel on dispose d'une solution analytique du problème pour un des critères de gestion, l'espérance de revenu. Pour obtenir cette solution analytique, il suffit de reprendre le raisonnement présenté dans Terreaux [11] concernant les incendies de forêts, dont le principe est inspiré de Reed [10]. Si l'on supprime cette hypothèse simplificatrice, alors il n'est malheureusement plus aussi 


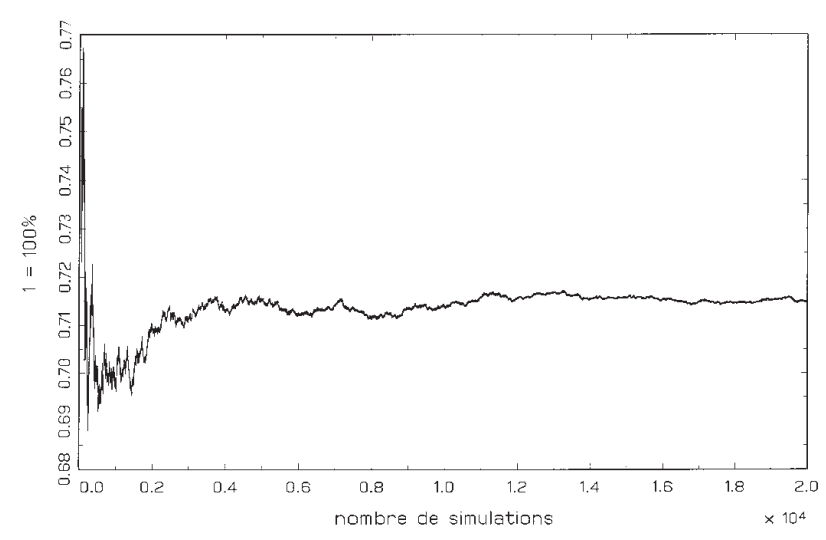

Figure 2. Eucalyptus gundal, durée de retour du gel de 30 ans, arbres coupés à 11 ans ; critère "probabilité d'avoir un gain supérieur à $30000 \mathrm{~F}$ », en fonction du nombre de simulations.

aisé d'avoir une solution analytique. De même, il n'est pas aussi direct d'avoir des résultats sur les autres critères de gestion retenus ici.

Enfin, en ce qui concerne toujours l'espérance de gain, une solution précise et sans la simplification précédente, a pu être obtenue en utilisant le fait que le système est d'évolution markovienne : le nombre $N$ d'états possibles de la parcelle est fini, et la probabilité de passage d'un état à un autre ne dépend que de l'état présent de la parcelle et pas de son histoire. En construisant des matrices de transfert d'un état à l'autre, de taille $N \times N$, nous avons obtenu une solution exacte concernant l'espérance de gain. Mais, par cette méthode, il n'est pas possible de déduire de ce type calculs la valeur des autres paramètres. Aussi ce résultat a été utilisé uniquement pour confirmer que la valeur obtenue après 20000 simulations par la méthode de Monte-Carlo est bien la limite de convergence quand le nombre de simulations tend vers l'infini.

3) En calculant une suite aussi grande de nombres pseudo-aléatoires, il était à craindre soit un recyclage de ces nombres (à savoir la répétition de suites identiques de périodes de gel et de non gel, qui n'ont plus alors les propriétés souhaitées), l'autocorrélation de la suite engendrée, ou encore l'obtention de nombres non équi-distribués (voir Vila [14]). On utilise ici un générateur congruentiel multiplicatif, c'est-à-dire du type :

$z_{i+1}=a z_{i}(\bmod . m)$, avec $z_{i}$ la suite de nombres générés, $i \in \mathfrak{N}, z_{1}$ choisi arbitrairement.

Les valeurs numériques utilisées ici sont $m=2^{31}-1$ et $a=397204094$.

Ce type de générateur a été initialement introduit par Lehmer [8]. Il présente bien les propriétés recherchées (Fishman [6]) et est d'un emploi particulièrement rapide (Vila [14]).

\section{LES RÉSULTATS}

\subsection{Résultats sur le gundal et sur le gunnii}

Pour une durée de retour du gel de 15 ans, les figures 3 et 4 présentent pour le gundal les valeurs des paramètres annoncés section 2.1 en fonction de l'âge de coupe des arbres.

Lorsque la durée de retour du gel est différente, et aussi pour le gunnii, les figures gardent la même allure, à savoir qu'elles indiquent que s'il est en général peu dommageable de retarder l'âge de coupe d'une ou de quelques

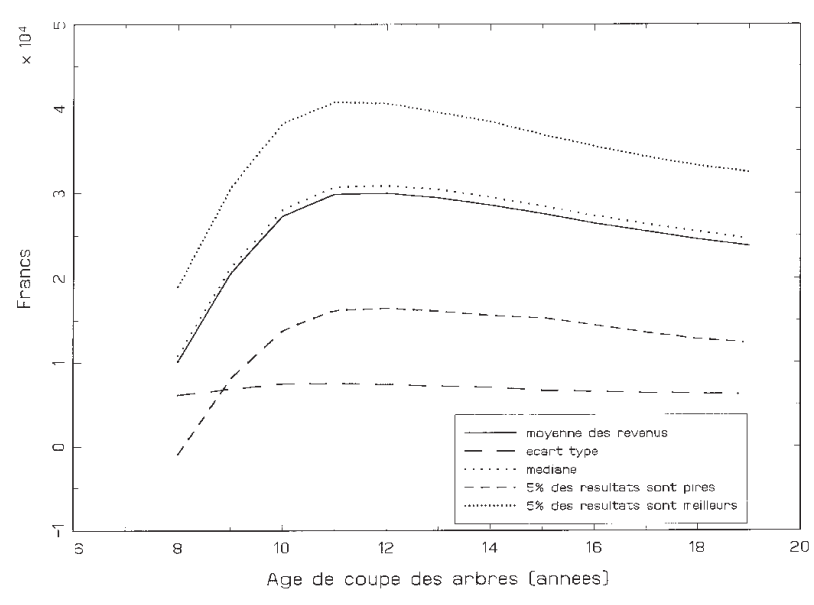

Figure 3. Eucalyptus gundal, durée de retour du gel de 15 ans : résultats économiques selon 5 critères, en fonction de l'âge de coupe des arbres.

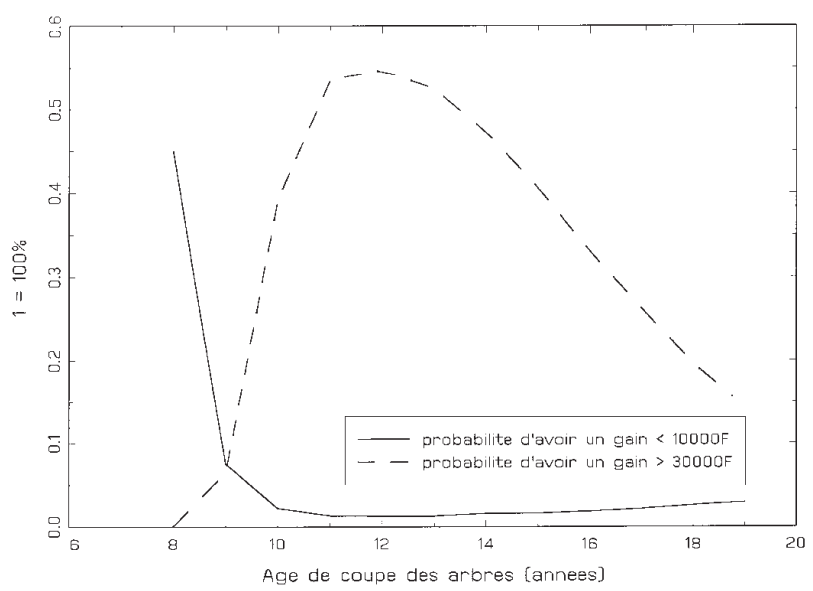

Figure 4. Eucalyptus gundal, durée de retour du gel de 15 ans : résultats économiques selon deux autres critères, en fonction de l'âge de coupe des arbres. 
Tableau I. Eucalyptus gundal : résultat des différents critères, pour les données indiquées en annexe. Les chiffres en italique correspondent à la valeur optimale, pour le critère considéré, de la date de coupe des arbres lorsqu'il n'y a pas gel.

GUNDAL :

\begin{tabular}{lcccccccccc}
\hline durée de retour du gel & 10 ans & 10 ans & 10 ans & 10 ans & 10 ans & 15 ans & 15 ans & 15 ans & 15 ans & 15 ans \\
\hline âge de coupe des arbres & 9 ans & 10 ans & 11 ans & 12 ans & 13 ans & 9 ans & 10 ans & 11 ans & 12 ans & 13 ans \\
\hline moyenne des revenus & 12478 & 18667 & 21245 & $\mathbf{2 1 6 6 9}$ & 21371 & 20536 & 27300 & 29893 & $\mathbf{3 0 0 0 7}$ & 29466 \\
écart type & $\mathbf{8 4 8 6}$ & 9087 & 9267 & 9197 & 9057 & $\mathbf{6 8 6 0}$ & 7465 & 7547 & 7396 & 7194 \\
médiane & 13214 & 19384 & 21944 & $\mathbf{2 2 4 9 9}$ & 22234 & 21202 & 28034 & 30715 & $\mathbf{3 0 8 7 7}$ & 30427 \\
$5 \%$ des résultats sont pires & -2661 & 2376 & 4769 & $\mathbf{5 3 5 2}$ & 5098 & 8057 & 13818 & 16191 & $\mathbf{1 6 4 2 9}$ & 16096 \\
$5 \%$ sont meilleurs & 25073 & 32265 & 35041 & $\mathbf{3 5 2 4 7}$ & 34683 & 30545 & 38208 & $\mathbf{4 0 7 5 2}$ & 40609 & 39539 \\
probabilité revenu < 10000 F & 35,75 & 16,87 & 11,77 & $\mathbf{1 0 , 9 1}$ & 11,48 & 7,54 & 2,17 & 1,30 & $\mathbf{1 , 2 1}$ & 1,26 \\
probabilité revenu > 30000 F & 0,49 & 9,77 & 17,67 & $\mathbf{1 8 , 7 3}$ & 17,30 & 6,52 & 39,33 & $\mathbf{5 3 , 6 4}$ & $\mathbf{5 4 , 6 6}$ & 52,45
\end{tabular}

GUNDAL :

\begin{tabular}{|c|c|c|c|c|c|c|c|c|c|c|}
\hline durée de retour du gel & 20 ans & 20 ans & 20 ans & 20 ans & 20 ans & 30 ans & 30 ans & 30 ans & 30 ans & 30 ans \\
\hline âge de coupe des arbres & 9 ans & 10 ans & 11 ans & 12 ans & 13 ans & 9 ans & 10 ans & 11 ans & 12 ans & 13 ans \\
\hline oyenne des revenus & 24408 & 31618 & 34212 & 34100 & 33177 & & 35862 & & 38073 & 36855 \\
\hline & & & & & & & & & & \\
\hline & & & & & & & & & & \\
\hline $\mathrm{s}$ résultats sont pires & 13486 & 19982 & 22247 & 22456 & 2181 & & & 28543 & 28528 & 27739 \\
\hline a & 32995 & 40617 & 43394 & 42880 & 41473 & 35039 & 42894 & 45354 & 44616 & 42939 \\
\hline probabilité revenu $<10000 \mathrm{~F}$ & 2,03 & 0,34 & 0,27 & 0,24 & 0,20 & 0,14 & 0,02 & 0,02 & 0,02 & 0,02 \\
\hline probabilité revenu > $30000 \mathrm{~F}$ & 17,86 & 63,91 & 76,24 & 76,57 & 73,56 & 43,18 & 86,32 & 92,77 & 92,68 & 90,73 \\
\hline
\end{tabular}

années, avancer cet âge éloigne sensiblement les critères de leur valeur optimale.

Les tableaux I et II présentent, pour le gundal puis pour le gunnii, en fonction de la durée de retour du gel et de l'âge de coupe des arbres, les valeurs prises par ces différents critères. On a indiqué les résultats pour des âges de coupe variant entre 9 et 13 ans seulement, puisque, hors de cette plage, quelque soit le risque de gel, aucun des critères indiqués (excepté la minimisation de l'écart type) ne prend sa valeur optimale.

\subsection{Résultats sur la diversification}

Les résultats précédents ont montré que le gundal est, pour un lieu donné, une essence beaucoup plus rentable mais aussi beaucoup plus risquée que le gunnii. La question qui s'en déduit naturellement, au niveau d'une propriété, est celle de l'intérêt éventuel de la diversification de la plantation entre ces deux essences.

On sait en effet qu'il est souvent judicieux de diversifier des investissements entre deux productions aux risques corrélés négativement : si la rentabilité est mau- vaise avec l'un, elle a plus de chances d'être bonne avec l'autre. On minimise ainsi le risque de perte.

Le problème se pose ici en des termes différents, puisque l'on peut considérer que si le gunnii gèle, alors le gundal gèle aussi. Il est cependant raisonnable de se poser la question de connaître s'il ne serait pas intéressant de consacrer une partie de la surface au gunnii, afin d'avoir avec une bonne probabilité un minimum de revenus, et d'utiliser le reste de la surface pour le gundal, pour tenter d'accroître les gains potentiels.

La propriété serait donc divisée en deux parties : une partie à haut risque et à haut rendement potentiel, une autre partie au contraire soumise à peu de risque, à rendement plus faible, et visant surtout à assurer un minimum de rentabilité.

La simulation qui suit se place dans ce cas de figure. Elle concerne une propriété pour laquelle la durée de retour du gel est :

- pour le gundal de 6 ans la première année de plantation, et de 10 ans les années suivantes,

- pour le gunnii : de 16 ans.

(Ces durées de retour correspondent à une localisation à Cugnaux en Haute-Garonne). 
Tableau II. Présentation identique des résultats de l’Eucalyptus gunnii.

GUNNII :

\begin{tabular}{lcccccccccr}
\hline durée de retour du gel & 10 ans & 10 ans & 10 ans & 10 ans & 10 ans & 15 ans & 15 ans & 15 ans & 15 ans & 15 ans \\
\hline âge de coupe des arbres & 9 ans & 10 ans & 11 ans & 12 ans & 13 ans & 9 ans & 10 ans & 11 ans & 12 ans & 13 ans \\
\hline moyenne des revenus & 1123 & 6497 & 8731 & 9374 & $\mathbf{9 5 5 7}$ & 8280 & 14122 & 16692 & $\mathbf{1 7 0 2 6}$ & 16893 \\
écart type & $\mathbf{7 5 4 0}$ & 8045 & 8330 & 8309 & 8157 & $\mathbf{6 1 3 0}$ & 6590 & 6674 & $\mathbf{6 6 1 7}$ & $\mathbf{6 4 8 2}$ \\
médiane & 1749 & 7099 & 9498 & 10123 & $\mathbf{1 0 4 1 2}$ & 8888 & 14837 & 17432 & $\mathbf{1 7 8 1 3}$ & 17724 \\
$5 \%$ des résultats sont pires & -12241 & -7771 & -6290 & -5648 & $\mathbf{- 5 2 0 4}$ & -2786 & 2233 & 4569 & 4887 & $\mathbf{4 9 3 4}$ \\
$5 \%$ sont meilleurs & 12409 & 18543 & 21165 & $\mathbf{2 1 6 7 2}$ & 21464 & 17141 & 23660 & 26302 & $\mathbf{2 6 4 1 2}$ & 26096 \\
probabilité revenu < 10000 F & 88,80 & 64,23 & 52,31 & 49,47 & $\mathbf{4 7 , 9 9}$ & 57,38 & 24,45 & 15,85 & 14,55 & $\mathbf{1 4 , 5 4}$ \\
probabilité revenu > 20000 F & 0,00 & 2,73 & 7,20 & $\mathbf{8 , 7 6}$ & 8,26 & 0,57 & 18,90 & 34,43 & $\mathbf{3 6 , 5 8}$ & 35,40 \\
\hline
\end{tabular}

GUNNII :

\begin{tabular}{|c|c|c|c|c|c|c|c|c|c|c|}
\hline durée de retour du gel & 20 ans & 20 ans & 20 ans & 20 ans & 20 ans & 30 ans & 30 ans & 30 ans & 30 ans & 30 ans \\
\hline âge de coupe des arbres & 9 ans & 10 ans & 11 ans & 12 ans & 13 ans & 9 ans & 10 ans & 11 ans & 12 ans & 13 ans \\
\hline moyenne des revenus & 11790 & 17924 & 20509 & 20732 & 20388 & 15385 & 21732 & 24273 & 24317 & 23636 \\
\hline écart type & 5305 & 5654 & 5855 & 5698 & 5448 & 4322 & 4648 & 4674 & 4516 & 4394 \\
\hline médiane & 12418 & 18561 & 21275 & 21550 & 21185 & 16033 & 22430 & 25009 & 25019 & 24399 \\
\hline $5 \%$ des résultats sont pires & 2151 & 7632 & 9759 & 10189 & 10229 & 7405 & 13065 & 15493 & 15727 & 15213 \\
\hline $5 \%$ sont meilleurs & 19318 & 25927 & 28741 & 28591 & 27868 & 21198 & 27931 & 30468 & 30173 & 29177 \\
\hline probabilité revenu $<10000 \mathrm{~F}$ & 33,01 & 9,04 & 5,26 & 4,73 & 4,72 & 11,47 & 1,84 & 0,83 & 0,73 & 0,89 \\
\hline probabilité revenu > $20000 \mathrm{~F}$ & 2,93 & 40,13 & 58,37 & 60,36 & 58,47 & 13,48 & 68,68 & 82,94 & 83,63 & 81,26 \\
\hline
\end{tabular}

On a alors comparé la plantation soit de 10 ha de gundal, soit de 10 ha de gunnii, soit de 5 ha de gundal et de 5 ha de gunnii. L'âge de coupe des arbres est de 12 ans pour les deux essences.

Les résultats (tableau III) sont présentés sous la même forme qu'auparavant, excepté pour les deux derniers critères, puisque les calculs concernent une plantation totale de 10 ha : on détermine respectivement la probabilité d'avoir un revenu inférieur à $100 \mathrm{kF}$ et supérieur à $200 \mathrm{kF}$. Pour des raisons similaires à celles données dans la section 2.2, on utilise ici 40000 simulations par type de plantation.
La diversification des investissements permet d'améliorer les résultats des critères « $5 \%$ des résultats sont pires » et «probabilité d'obtenir un revenu inférieur à $100 \mathrm{kF} \gg$. Cela représente en fait une diminution du risque d'avoir de faibles revenus.

Par rapport à la plantation du seul gunnii, la diversification permet d'améliorer les valeurs prises par les différents critères, sauf celle de l'écart type des revenus, alors que remplacer l'intégralité des E. gunnii par des E. gundal augmenterait le risque d'avoir de faibles revenus (critères « $5 \%$ des résultats sont pires » et «probabilité d'avoir un résultat $\langle 100 \mathrm{kF} \gg$ ) ainsi bien sûr que la dispersion des résultats (écart type nettement plus élevé).

Tableau III. Présentation des résultats de la plantation de 10 ha d'eucalyptus à Cugnaux (Haute-Garonne) avec et sans diversification entre gundal et gunnii.

\begin{tabular}{|c|c|c|c|}
\hline diversification ou non & 10 ha de gundal & 5 ha de gundal et 5 ha de gunnii & 10 ha de gunnii \\
\hline moyenne des revenus & $211540 \mathrm{~F}$ & $195860 \mathrm{~F}$ & $180020 \mathrm{~F}$ \\
\hline écart type & $92844 \mathrm{~F}$ & $72755 \mathrm{~F}$ & $63939 \mathrm{~F}$ \\
\hline médiane & $219960 \mathrm{~F}$ & $202940 \mathrm{~F}$ & $187900 \mathrm{~F}$ \\
\hline $5 \%$ des résultats sont pires & $45579 \mathrm{~F}$ & $63762 \mathrm{~F}$ & $62180 \mathrm{~F}$ \\
\hline $5 \%$ sont meilleurs & $348180 \mathrm{~F}$ & $302720 \mathrm{~F}$ & $270230 \mathrm{~F}$ \\
\hline probabilité revenu < $100000 \mathrm{~F}$ & $12,19 \%$ & $10,22 \%$ & $11,46 \%$ \\
\hline probabilité revenu > $200000 \mathrm{~F}$ & $58,39 \%$ & $51,63 \%$ & $42,22 \%$ \\
\hline
\end{tabular}




\section{DISCUSSION}

Étant donné que les sols utilisables pour entreprendre une culture de l'eucalyptus peuvent être de mauvaise qualité agronomique, donc de faible valeur marchande, il n'est pas déraisonnable d'utiliser l'Eucalyptus gundal dès que la durée de retour du gel dépasse dix ans pour la ou les parcelles considérées. Le tableau I donne des indications pour la détermination de l'âge de coupe. L'Eucalyptus gunnii, quant à lui, procure des résultats plus réguliers mais moins bons.

En ce qui concerne l'âge de coupe, les figures 3 et 4 , qui concernent le gundal soumis à une durée de retour du gel de 15 ans, montrent qu'il est pour la plupart des critères peu dommageable de retarder l'âge de la récolte. Il est moins raisonnable de l'avancer. Cette conclusion reste valable pour d'autres durées de retour du gel ainsi que pour le gunnii, les courbes les concernant gardant la même allure.

Ces résultats dépendent fortement des données utilisées et ne peuvent être généralisés sans précaution. De plus ils ont un caractère statistique, c'est-à-dire qu'il s'agit de moyennes obtenues sur un grand nombre de réalisations. Il n'est pas impossible que les eucalyptus aient à subir, dès les premières années, une succession de gels, ce qui pourrait amener à regretter a posteriori ce choix d'investissement, et cela quelle que soit la durée de retour du gel. On veillera donc à ne pas interpréter abusivement ces résultats quantitatifs, et à ne pas utiliser ces chiffres comme des garanties de résultats.

On notera que l'on n'a pas tenu compte du coût induit par l'irréversibilité de telles plantations, en particulier lorsqu'on les compare à des cultures annuelles : les résultats présentés ici tiennent compte du fait que l'on recèpe au moins deux fois les arbres, donc, pour des rotations de 12 ans, que l'on mobilise le sol pour les 36 années à venir.

Mais inversement, pour un agriculteur, il est certain que la charge de travail induite directement ou indirectement par une telle production est beaucoup plus faible que celle induite par la culture de plantes annuelles. L'eucalyptus peut donc lever certaines contraintes, en particulier en cas d'agrandissement de la surface de l'exploitation, de diminution de la quantité de main d'œuvre disponible, de préparation à la retraite (notion de constitution d'un patrimoine), tout en procurant des revenus nettement plus proches et réguliers qu'une sylviculture traditionnelle.

Remerciements : Je tiens à remercier S. Viéban de la SEBSO, l'équipe de l'Afocel, station de Cugnaux, qui a fourni les données permettant de mener à terme ce travail, et plus particulièrement MM B. Cauvin et P. Burger Leenhardt pour leurs fructueuses suggestions. Je remercie aussi J.C. Hervé pour ses précieux conseils qui ont permis d'améliorer considérablement la qualité du manuscrit. Ces travaux ont été financés en partie grâce au contrat de plan État-Région Midi-Pyrénées 1994-1998.

\section{RÉFÉRENCES}

[1] Bouzit M., Modelling farmers' behaviour under risk via anticipated utility maximisation, Working Paper 97-03, Cemagref, Division Irrigation, Montpellier, 1997.

[2] Brumelle S., Stanbury W.T., Thompson W.A., Vertinsky I., Wehrung D., Framework for the analysis of risks in forest management and sylvicultural investments, Forest Ecol. manage. 35 (1990) 279-299.

[3] Cauvin B., Melun F., Guide de culture du TCR d'eucalyptus, Afocel Armef, Informations forêt n 3486 (1994) 205224.

[4] Cauvin B., Bonduelle P., Hubert Cl., Les taillis à courte rotations : une culture pour la jachère fixe, Afocel-Armef Informations-forêt, $n^{\circ} 1475$ (1994) 21-39.

[5] Faustmann M., 1849, Calculation of the value which forest land and immature stands possess for forestry, reprint in J. For. Econom. 11 (1995) 7-44.

[6] Fishman G.S., Monte Carlo, concepts algorithms and applications, Springer, New York, 1995.

[7] Hammersley J.M., Handscomb D.C., Les méthodes de Monte Carlo, Dunod, Paris, 1967.

[8] Lehmer D.H., Mathematical methods in large-scale computing methods, Ann. Comp. Lab., Harvard University 26 (1951) 141-146.

[9] von Neumann J., Morgenstern O., Theory of games and economic behavior, Princeton University Press, Princeton, N.J., 1954.

[10] Reed W.J., The effects of the risk of fire on the optimal rotation of a forest, J. Envir. Econom. Manage. 11 (1984) 180190.

[11] Terreaux J.P., Principes de gestion des investissements en forêt, Thèse de doctorat nouveau régime, Université de Toulouse I, 1990.

[12] Terreaux J.P., 1995, Gestion et évaluation des forêts : éléments pour le choix d'un taux d'actualisation, Séminaire du Groupe de Recherche en Économie des Produits Forestiers, «Monnaie, finance et filière forêt-bois-papier », 29 Juin 1995, Bordeaux.

[13] Ventsel H., Théorie des probabilités, Ed. MIR, Moscou, 1973.

[14] Vila J.P., Construction d'un pseudo-hasard, cours de DEA, INRA - biométrie, Montpellier, 1997. 


\section{ANNEXE}

\section{Les données}

Les données de cette section concernent une parcelle de un hectare d'Eucalyptus gundal ou gunnii. Les valeurs sont données en francs français. Leur source est l'Association Forêt Cellulose (AFOCEL), ainsi que Cauvin B. \& Melun F. (1994) et Cauvin B., Bonduelle P. \& Hubert Cl. (1994).

Production cumulée moyenne en tonnes vertes :

\begin{tabular}{rcc}
\hline âge & gunnii & gundal \\
\hline 1 & 0 & 0 \\
2 & 0 & 0 \\
3 & 0 & 0 \\
4 & 20 & 25 \\
5 & 37 & 46 \\
6 & 58 & 72 \\
7 & 83 & 104 \\
8 & 116 & 145 \\
9 & 151 & 189 \\
10 & 182 & 228 \\
11 & 203 & 254 \\
12 & 215 & 269 \\
13 & 224 & 280 \\
14 & 231 & 289 \\
15 & 236 & 295 \\
\hline
\end{tabular}

-Frais de culture :

- frais d'implantation : $11000 \mathrm{~F}$ (première implantation, ou lorsque l'on a épuisé les possibilités de recépage);
- frais de recépage : $1800 \mathrm{~F}$;

- Prix de vente du bois (sur pied) : $66 \mathrm{~F} /$ tonne verte (par contrat avec l'industrie utilisatrice, cette valeur est réactualisée pour tenir compte de l'inflation éventuelle. Ici nous ne tenons compte ni de cette réactualisation, ni de l'inflation).

-Taux d'actualisation utilisé : $2 \%$ (voir Terreaux [12]);

-La parcelle peut être recépée deux fois de suite, et deux fois seulement;

- Si la parcelle gèle, les arbres sont récoltés, et le volume qu'ils représentent (voir tableau précédent) est commercialisé, sans décote au niveau des prix ;

- Les arbres sont plantés au printemps et s'il y a gel dès le premier hiver, les arbres se recèpent naturellement, sans intervention du sylviculteur. Il n'y a donc pas de frais de recépage, mais on ne pourra les recéper qu'une seule fois par la suite;

-Nous ne tenons pas compte ici des aides publiques éventuelles qui pourraient être attribuées à ce type de plantation.

Durée de retour du gel : on considère ici des durées de retour du gel comprises entre 10 et 30 ans. Cette durée correspond à l'intervalle de temps moyen séparant deux événements gel entraînant la mort des arbres. Si deux tels événements ont lieu le même hiver, ils ne sont comptés que comme un seul.

Enfin on rappelle que le gundal étant beaucoup plus gélif que le gunnii, pour une parcelle donnée, les périodes de retour d'un gel destructeur sont sensiblement plus faibles pour le premier que pour le second. 